\title{
The deleterious effects of chronic obstructive pulmonary disease and obstructive sleep apnea: pathophysiology and implications on treatment
}

Ahmed Gharibi

\begin{abstract}
Background: Chronic obstructive pulmonary disease (COPD) and obstructive sleep apnea (OSA) are both common in the adult population. The coexistence and association of both COPD and OSA have been described as the overlap syndrome. The scope of the present review is to address the magnitude, pathophysiology, clinical presentations, and the deleterious consequences of the coexistence of COPD and OSA in the same patient as well as the current management and treatment options of this association.

Main body of abstract: Epidemiological studies showed a prevalence of the overlap syndrome of up to $1 \%$ of the adult population. However, various studies agreed that this association is due to the fact that both COPD and OSA are common rather than an interaction of pathophysiology between the two affections. Nevertheless, sleep may cause adverse effects on breathing and lung functions. While these effects can be neglected in otherwise healthy individuals, they can lead in contrast to disastrous clinical outcomes particularly in vulnerable patients with the overlap syndrome leading to increased risk of cardiovascular diseases, acute COPD exacerbations, increased rates of hospitalizations, and nocturnal deaths. Current guidelines recommend polysomnography (PSG) as the gold standard investigation to diagnose sleep disorders and overlap syndrome. Treatment may include pulmonary rehabilitation, supplemental oxygen therapy, and non-invasive ventilation. Currently, continuous positive airway pressure (CPAP) therapy is the treatment of choice for the overlap syndrome. CPAP effects include improved respiratory mechanics; sleep quality, exercise tolerance, and prolonged survival.
\end{abstract}

Conclusion: Both COPD and OSA are common in the general population and present a significant risk of increased morbidity and mortality when they coexist in the same patient. Clinicians must carefully evaluate the clinical outcomes and the high risk of cardiovascular complications related to the overlap syndrome. Current data indicate that CPAP treatment leads to amelioration of the health-related quality of life and improve survival in patients with the overlap syndrome.

Keywords: Obesity, COPD, OSA, CPAP

Correspondence: ahmedgharib@hotmail.com

Pulmonary Research Unit, Department of Internal Medicine, National

Research Centre, 36 Abdel Khalek Tharwat St, Downtown, Cairo, Egypt

\section{Springer Open}

( ) The Author(s). 2020 Open Access This article is licensed under a Creative Commons Attribution 4.0 International License, which permits use, sharing, adaptation, distribution and reproduction in any medium or format, as long as you give appropriate credit to the original author(s) and the source, provide a link to the Creative Commons licence, and indicate if changes were made. The images or other third party material in this article are included in the article's Creative Commons licence, unless indicated otherwise in a credit line to the material. If material is not included in the article's Creative Commons licence and your intended use is not permitted by statutory regulation or exceeds the permitted use, you will need to obtain permission directly from the copyright holder. To view a copy of this licence, visit http://creativecommons.org/licenses/by/4.0/. 


\section{Background}

Chronic obstructive pulmonary disease (COPD) and obstructive sleep apnea (OSA) are both common in the adult population. It is still unclear if each disease increases the odds of incidence of the other or they coexist together simply because of their high prevalence [1]. The term overlap syndrome (OS) has been used to refer to the presence of COPD and OSA in the same patient [2].

COPD should be considered in patients presented with a history of tobacco smoking and symptoms of dyspnea, chronic cough, or sputum production. Pulmonary function measurements of pre- and post-bronchodilator inhalation are used to affirm the extent of the disease and assess the degree of airflow obstruction defined as $\mathrm{FEV}_{1} /$ FVC $<0.7$ and $\mathrm{FEV}_{1}<80 \%$ predicted. According to the current GOLD guidelines, COPD is characterized by persistent airway obstruction as post-bronchodilator $\mathrm{FEV}_{1} /$ FVC remains $<0.7$ [3]. COPD is also known to be a major cause of the increased risk of death worldwide with a global prevalence of around 12\% [4]. In a study published in 2015, Said et al. reported that COPD was present in $10 \%$ of a high-risk group of Egyptian patients [5].

COPD is also known to evolve on a background of local and systemic inflammation carrying considerable morbidity and mortality. Interestingly, studies have shown increased nocturnal mortality during COPD exacerbations with around $70 \%$ of deaths occurring between midnight and 08:00 am [6]. In addition, patients with COPD are at increased risk of developing cardiovascular diseases and around 50\% of patients die from cardiovascular complications [7].

On the other hand, OSA is characterized by episodes of complete or partial obstruction of the upper airway leading to intermittent hypoxia, frequent arousals, and daytime sleepiness. The severity of OSA is assessed with the apnea-hypopnea index (AHI), the number of apneas and hypopneas per hour of sleep, categorized into: mild (AHI 5-15), moderate (AHI 15-30), and severe (AHI >30) [8]. The prevalence of OSA has been investigated in different western population cohorts, varying around $34 \%$ for men and $17 \%$ for women. This incidence of OSA is expected to rise even more with the explosion of the obesity epidemics around the world $[9,10]$.

A recent study that included around 1000 patients referred for polysomnography in Cairo, Egypt, found that $83 \%$ of the patients were diagnosed with OSA, agreeing with similar results elsewhere in Egypt [11-14]. Even though these studies were retrospective, single centered, and their results cannot be projected on the entire population but at least confirming that OSA is the most common sleep disorder encountered in hospitals and clinics around Egypt and special attention must be given to it.

The OS is considered when a clinical history of COPD or emphysema, FEV1/FVC less than $70 \%$, and a definite diagnosis of OSA are all present together [2]. Whether COPD predisposes to OSA or vice versa due to a causal link of complex pathophysiology that might exist between these two affections is still unknown.

It has been demonstrated that cigarette smoking significantly increased edema and inflammation leading to narrowing of the upper airways. For this reason, tobacco smoking has been considered a contributing factor to develop OSA in patients with COPD [15]. Also, it is well proved that the pharyngeal collapse in OSA is related to central obesity which is further promoted in patients with COPD due to their wide use of corticosteroids [16]. Other well-known risk factors that increase the risk of OSA include obesity, age, male gender, and upper airway anatomical abnormalities [17].

However, it is fair to think that the coincidence of COPD and OSA in the same patient is somehow expected and is probably due to chance alone since both diseases are very common in the general population. Further studies are needed to fully understand this relationship and to determine the prevalence of the OS.

\section{Main text \\ Epidemiology}

The prevalence of OS remains challenging. Results from the Sleep Heart Health Study that included almost 6000 patients have shown that the coexistence of both of these conditions is not rare in clinical settings and far from negligible. In fact, according to European cohorts, the OS can be observed in $1 \%$ of adult patients $[18,19]$. A higher prevalence was found in a Korean study. The authors evaluated about 1300 patients with spirometry and laboratory polysomnography (PSG) and found that the overlap existed in $5 \%$ of this adult cohort [20].

In contrast, other studies investigated the prevalence of the OS in OSA and COPD respectively with highly variable prevalence figures reported depending on the diagnostic techniques employed but with more than half of patients with COPD were found to have associated OSA $[21,22]$. In a cross-sectional study on a large number of patients, the OS was the final diagnosis in $12 \%$ of patients diagnosed with OSA [23].

Regarding the OS in Egypt, to the present author's knowledge, only three studies while assessing comorbidities associated with OSA noted different incidence figures of COPD among their patients varying between 4 , 16 , and $57 \%[13,24,25]$.

\section{Pathophysiology}

Sleep leads to reduced respiratory drive, functional residual capacity (FRC), and skeletal muscle activity. These normal physiological respiratory responses that occur during sleep are frequently accentuated in patients diagnosed with COPD, particularly during the rapid eye 
movement (REM) stage of sleep. During REM, reduced tidal volume, and diminished respiratory drive contribute to an insignificant fall in arterial oxygen saturation in healthy subjects [26].

However, this response is amplified in patients with COPD with limited oxygen reserves and respiratory muscle weakness, aggravating the hypoxemia and/or hypercapnia, normally present during wakefulness in those patients. As a consequence, patients with COPD are subjects to severe complications and a high risk of mortality at night during acute exacerbations [27, 28].

Furthermore, during sleep, the supine position has a negative impact on patients with COPD. This position is associated with the collapse of the small airways in dependant lung zones as well as a marked decrease in the FRC of the lungs leading to deterioration in gas exchange in COPD. In addition, the rostral fluid shift from peripheral edema when supine result in fluid accumulation around the neck favoring upper airway obstruction and the onset of obstructive respiratory events during the night [29].

It is worth to mention that COPD is a spectrum of clinical phenotypes predominantly emphysema or a chronic bronchitis phenotype. The association of OSA with the emphysema phenotype may be low as recent evidence showed that gas trapping lowers the pharyngeal critical closing pressure during sleep thus protective from OSA. The extent of hyperinflation assessed by CT scans was shown to correlate negatively and significantly with the severity of OSA during REM stages in a recent study conducted on patients diagnosed with severe COPD but the authors failed to show the same correlation during non-REM sleep stages [22].

Conversely, the predominant chronic bronchitis phenotype promotes the development of OSA for several reasons: higher BMI, chronic use of steroids that may increase neck size, higher prevalence of cor pulmonale, increased likelihood of pharyngeal collapse due to edema or muscle weakness leading to easy upper airway collapsibility. Lastly, patients with chronic bronchitis are more prone to gas exchange abnormalities due to the associated decrease in their respiratory drive [30].

On a parallel note, systemic inflammation as well as the release of various inflammatory mediators is closely linked to both COPD and OSA. A wide number of inflammatory pathways were studied and reported to be key factors in the progression of both diseases [31]. Also, the disturbance in gas exchange in patients with COPD induces oxidative stress with high levels of C-reactive protein and interleukin- 6 routinely found in their BAL [32]. Smoking further exacerbates the underlying inflammatory process leading to deterioration in the pathophysiological pathways [33].
In accord with the inflammatory process, reactive oxygen species are also highly expressed in patients with OSA mostly as a result of the intermittent hypoxemia encountered in those patients [34]. This in turn will affect various cellular components and therefore, it is no surprise that patients with OSA are subjects to vascular endothelial dysfunction [35].

Consequently, due to the inflammatory process described in patients with COPD and OSA, they are at increased risk of progression to atherosclerosis and cardiovascular diseases [36]. Additionally, it was demonstrated that patients with the OS have increased sympathetic activity, which makes them more liable for cardiac complications [37]. Hence, the inflammatory and oxidative stress pathways together can be critical to the serious clinical consequences observed in COPD and OSA when they coexist in the same patient [38].

Clearly, sleep has deleterious effects on breathing, respiratory drive, and functions of the lung. While these effects can be neglected in otherwise healthy individuals, they can lead in contrast to disastrous clinical outcomes particularly in vulnerable patients with the overlap syndrome. These patients may experience pronounced hypoxemia and hypercapnia, reduced quality of life, and increased risk of death [39]. The latter, frequently reported during the night, mainly due to ventilationperfusion mismatching and alveolar hypoventilation [40]. These mechanisms are all negatively impacted by the effect of obesity that further reduces lung mechanics and the strength of the respiratory muscle [41].

\section{Clinical and cardiovascular consequences}

Both diseases are characterized by severe clinical symptoms and comorbidities. No doubt, we could only assume that patients with the OS will present with aggravated symptoms and worse prognosis. Patients with OS often report disturbed sleep rhythm, daytime fatigue, and sleepiness. Biswas et al. showed that those patients describe significantly worse quality of life that is usually accompanied by anxiety and depression [42] .

Noteworthy, a recent work noted that sleep impairment in COPD is associated with rapid reductions in pulmonary functions as well as daytime activity levels. Also, sleep quality and sleep architecture were negatively affected in patients with COPD [43]. Recently, poor sleep quality predicted deteriorated health-related quality of life and lead to adverse outcomes such as acute exacerbation, high hospitalization rates and costs particularly in patients with COPD $[44,45]$.

In fact, the presence of the OS was associated with a high frequency of hospitalizations from COPD exacerbations, severe respiratory symptoms, and low quality of life. Thus, increasing all-cause mortality compared with those with just COPD alone [46, 47]. Also, the incidence 
of acute respiratory failure in patients diagnosed with the OS is increased compared with either disease alone and correlated to nocturnal hypoxemia particularly in obese individuals. Therefore, the OS was previously considered a major cause of admission to the ICU [48].

On top of that, as previously discussed, it is possible that the coexistence of both COPD and OSA may worsen the inflammatory process, inducing a severe cardiovascular impairment. Of note, the association between OSA and cardiac diseases has been thoroughly reviewed and various results have demonstrated that patients with OSA are at high risk for systemic hypertension, coronary artery disease, and right-side heart failure [49].

Not surprisingly, the data from a European cohort reported that patients with the OS died often from cardiovascular complications [50]. Additionally, nearly $80 \%$ of the subjects with the OS were diagnosed with pulmonary hypertension $(\mathrm{PH})$ in comparison to around $15 \%$ of the patients who only had OSA [51].

Chaouat et al. observed that even without a severe degree of bronchial obstruction, patients with the overlap syndrome exhibited a high risk of developing respiratory insufficiency and $\mathrm{PH}$. In this study, patients with the OS had worse hypoxemia and hypercapnia compared with the whole population and nearly half of them were diagnosed with $\mathrm{PH}$ compared with only $13 \%$ in the rest of patients [52].

In a recent study, authors from China examined 106 patients with COPD and found that pulmonary artery pressure (PAP) was increased by $5 \mathrm{mmHg}$ in the group of patients with the OS (52.8\%) compared with COPD only patients. The increase in PAP positively correlated with reduced mean $\mathrm{SpO}_{2}$ levels during the night [21].

In addition, COPD has a significant risk of premature ventricular contractions while patients with OSA often present with atrial fibrillation. In fact, the latter is observed commonly and frequently in patients with the OS as opposed to either OSA or COPD alone according to a retrospective study that included 2873 patients [53].

Actually, authors from the Rotterdam study confirmed an increased risk of sudden cardiac death in patients with COPD. In this study, it was not mentioned if OSA coexisted in their patients; however, death occurred frequently during the night [54]. Thus, it is safe to assume that patients with the OS are at even greater risk since the predisposing factors for sudden cardiac death including ventricular arrhythmias, prolonged QT interval, gas exchanged abnormalities, systemic inflammation, and reduced respiratory drive during sleep are commonly present in patients with COPD and OSA.

\section{Diagnosis}

In clinical practice, screening for patients with OS should be done when: 1 -patients are presented with mild COPD but with evidence of $\mathrm{PH}$ or daytime hypercapnia disproportionate to the respiratory impairment. 2-COPD patients complaining of morning headaches despite treatment. 3-Patients with symptoms suggestive of OSA along with clinical and spirometry diagnosis of COPD [55].

Therefore, a history of sleep symptoms should be part of the routine clinical evaluation for COPD patients. Most common symptoms are snoring, fragmented sleep, gasping and choking during the night, nocturia, and increased daytime sleepiness. Also, overnight oximetry showing a typical sawtooth pattern during the night could be helpful to decide further clinical investigations [56].

The gold standard method for the diagnosis of the OS remains polysomnography (PSG) studies [57]. The American Thoracic Society/European Respiratory Society task force guidelines recommend overnight testing in patients with $\mathrm{PH}$ associated with mild COPD [58]. Although, no significant differences regarding the AHI between patients with the OS compared with OSA only patients in many PSG studies, the former group exhibited severe oxygen desaturations, significantly higher total sleep time spent with $\mathrm{SpO} 2$ less than $90 \%$ and reduced sleep efficiency (\% of sleep time) $[19,51,52]$.

However, due to the limited availability and the high cost of PSG, careful evaluation of suspected patients is required. Thus, questionnaires such as the Stop-BANG or the Epworth Sleepiness Scale (ESS) were developed to screen and determine the probability of OSA but they are not accurate enough to confirm or rule out OSA and replace PSG [59].

Further, Soler et al. demonstrated that in advanced COPD, the performance of the STOP-Bang even though was less than expected was associated with OSA to the contrary of the ESS. The authors also noted that in COPD patients, neck circumference was not a good predictor of OSA, indicating the presence of OSA in patients with advanced COPD even among those with small neck circumference [60].

Hence, despite limited data, the Stop-BANG, which consists of 8 (yes or no) questions and has the advantage of being easily scored, may serve as a screening tool in the initial evaluation of probable OSA in patients with COPD along with history and physical examination.

\section{Treatment}

Treatment objectives of the OS are directed towards the restoration of adequate oxygen saturation during sleep, reduce obstructive respiratory events, improve gas exchange, and undoubtedly improve sleep quality and the health-related quality of life in those patients. Treatment options include bronchodilators, pulmonary rehabilitation, long-term oxygen therapy (LTOT), and noninvasive ventilation (NIV). Equally important to these 
modalities is the reinforcement of sleep hygiene and weight loss measures. Also, sleep deprivation, alcohol, hypnotic drugs should be always discouraged in those patients.

Pulmonary rehabilitation including smoking cessation and structured exercise programs was shown to have a positive impact on patients. In fact, after 8 weeks of the pulmonary rehabilitation program, patients with COPD reported amelioration in sleep quality, less dyspnea, and reduced hospital visits with subsequent improvement in the quality of life [45].

In line with the above, numerous authors also reported that LTOT extended to sleeping hours may have beneficial effects on daytime and nocturnal hypoxemia. LTOT was associated as well with reduced risk of mortality in patients with COPD [61]. However, a randomized controlled study concluded that oxygen therapy should not be considered a definite treatment option in patients with OS as it has no role in preventing obstructive respiratory events during sleep [62].

Although CPAP is not considered to be a form of NIV in the strict sense, yet, CPAP remains the cardinal treatment modality and serves as an extremely practical measure to reverse the side effects of the OS. Marin et al. analyzed CPAP efficacy after a median follow-up of about 9 years. They found out that treatment with CPAP not less than $4 \mathrm{~h} /$ night reduced the AHI thereby improving oxygen saturation during sleep and was associated with decreased hospitalizations and risk of death [50].

Machado et al. evaluated treatment outcomes in 95 patients diagnosed with the overlap syndrome. Patients had moderate to severe OSA and were receiving LTOT for their COPD. In the CPAP-treated group, the 5-year survival estimate was $70 \%$ compared with $25 \%$ in the non-treated groups. After adjusting for several confounders, the study showed that patients treated with CPAP showed a significantly lower risk of death [63].

Moreover, de Miguel et al. observed that after 6 months of CPAP therapy in patient with the OS, lung functions and gas exchange parameters were significantly improved with a marked reduction in hypercapnia [64]. These results agreed with what was previously published after 3 months of CPAP treatment in a small number of patients with the OS. The improvement in lung function and gas exchange was indeed associated with a considerable fall in the risk for hospitalizations [65].

The positive effects of CPAP are mostly due to the improvement in respiratory mechanics. The use of CPAP is associated with increased functional residual capacity, improved lung function, ventilation-perfusion matching, and respiratory muscle strength [64]. Equally, CPAP may be effective in unloading inspiratory muscles and reducing work of breathing, thus, improves skeletal muscle strength leading to enhanced exercise tolerance
[66]. The hemodynamic benefits of CPAP also play an important role in reducing mortality due to cardiovascular diseases in patients with OSA [67]. Indeed, CPAP therapy has been coined with prolonged survival and better health-related quality of life in patients with OS $[63,68]$

\section{Conclusion}

COPD and OSA are both highly prevalent disorders. However, many clinicians should bear in mind that OSA leads to significantly damaging consequences on the quality of sleep and the pulmonary functions of subjects diagnosed with COPD. Clinicians should also be aware of the increased threat of cardiovascular complications encountered when COPD and OSA coexist in the same patient.

As a consequence, patients presented with symptoms related to OSA and COPD should be thoroughly evaluated and subjects to PSG studies. Simultaneous treatment of COPD and OSA is necessary to reduce the morbidity and mortality associated with the OS. It is well evidenced that the appropriate use of CPAP treatment leads to significant benefits to physical health, well being, and long-term survival in patients with the OS.

More work still needs to be done to accurately assess the role of screening and the diagnosis of OSA in patients with COPD and future research is essential to fully understand the complex interaction between COPD and OSA and to evaluate the clinical outcomes and complications of both diseases when they exist together, particularly regarding their potential influence on the frequency of COPD exacerbations, health-related quality of life and mortality.

\section{Abbreviations}

AHI: Apnea-hypopnea index; BAL: Broncho-alveolar lavage; COPD: Chronic obstructive pulmonary disease; CPAP: Continuous positive airway pressure; ESS: Epworth Sleepiness Scale; FEV1/FVC: Forced expiratory volume in 1st second/ forced vital capacity; FRC: Functional residual capacity; LTOT: Longterm oxygen therapy; NIV: Non-invasive ventilation; OS: Overlap syndrome; OSA: Obstructive sleep apnea; PAP: Pulmonary artery pressure;

PH: Pulmonary hypertension; PSG: Polysomnography; REM: Rapid eye movement; $\mathrm{SpO}_{2}$ : Peripheral capillary oxygen saturation

\section{Author's contributions}

AG contributed to the literature search, manuscript preparation, review and editing, guarantor. The author(s) read and approved the final manuscript.

\section{Availability of data and materials \\ Not applicable.}

Ethics approval and consent to participate

Not applicable.

Consent for publication

Not applicable.

Competing interests

The author declares that he has no competing interests. 
Received: 14 May 2020 Accepted: 21 August 2020 Published online: 01 September 2020

\section{References}

1. Kouismi H, El Ftouh M, Naji-Amrani H, El Fihry MTEF (2013) Overlap syndrome: association of chronic obstructive pulmonary disease and obstructive sleep apnea syndrome. Egypt J Chest Dis Tuberc. 62(4):583-587

2. Flenley DC (1985) Sleep in chronic obstructive lung disease. Clin Chest Med. 6(4):651-661

3. Singh D, Agusti A, Anzueto A, Barnes PJ, Bourbeau J, Celli BR et al (2019) Global strategy for the diagnosis, management, and prevention of chronic obstructive lung disease: the GOLD science committee report 2019. Eur Respir J. 53(5)

4. Adeloye D, Chua S, Lee C, Basquill C, Papana A, Theodoratou E et al (2015) Global and regional estimates of COPD prevalence: Systematic review and meta-analysis. J Glob Health. 5(2):020415

5. Said AF, Ewis AA, Omran AA, Magdy ME, Saleeb MF (2015) Prevalence and predictors of chronic obstructive pulmonary disease among high-risk Egyptians. Egypt J Bronchol 9:27-23

6. Owens RL, Macrea MM, Teodorescu M (2017) The overlaps of asthma or COPD with OSA: A focused review. Respirology. 22(6):1073-1083

7. Huiart L, Ernst P, Suissa S (2005) Cardiovascular morbidity and mortality in COPD. Chest. 128(4):2640-2646

8. Kapur VK, Auckley DH, Chowdhuri S, Kuhlmann DC, Mehra R, Ramar K et al (2017) Clinical practice guideline for diagnostic testing for adult obstructive sleep apnea: an American Academy of sleep medicine clinical practice guideline. J Clin Sleep Med. 13(3):479-504

9. Javaheri S, Barbe F, Campos-Rodriguez F, Dempsey JA, Khayat R, Javaheri S et al (2017) Sleep Apnea: types, mechanisms, and clinical cardiovascular consequences. J Am Coll Cardiol 69(7):841-858

10. Peppard PE, Young T, Barnet JH, Palta M, Hagen EW, Hla KM (2013) Increased prevalence of sleep-disordered breathing in adults. Am J Epidemiol. 177(9):1006-1014

11. Gharib A, Saleh A, Elbalsha A, Loza S (2019) An insight on the magnitude of obstructive sleep apnea in egypt: prevalence, gender differences and factors affecting apnea hypoapnea index severity. Sleep Med. 64:S128

12. El-Sayed IH (2012) Comparison of four sleep questionnaires for screening obstructive sleep apnea. Egypt J Chest Dis Tuberc. 61(4):433-441

13. Yousif M, El-Helbawy R (2013) A six-year sleep lab experience in an Egyptian University Hospital. Egypt J Chest Dis Tuberc. 62(4):717-722

14. Suliman L, Shalabi N, Elmorsy A, Moawed M (2017) Value of STOP-BANG and Berlin questionnaires in the diagnosis and severity prediction of obstructive sleep apnea hypopnea syndrome. Egypt J Bronchology. 11:367

15. Steveling EH, Clarenbach CF, Miedinger D, Enz C, Dürr S, Maier S et al (2014) Predictors of the overlap syndrome and its association with comorbidities in patients with chronic obstructive pulmonary disease. Respiration. 88(6):451457

16. Teodorescu M, Consens FB, Bria WF, Coffey MJ, McMorris MS, Weatherwax $\mathrm{KJ}$ et al (2009) Predictors of habitual snoring and obstructive sleep apnea risk in patients with asthma. Chest. 135(5):1125-1132

17. Deng X, Gu W, Li Y, Liu M, Li Y, Gao X. Age-Group-Specific Associations between the severity of obstructive sleep apnea and relevant risk factors in male and female patients. PLoS One [Internet]. 201411 [cited 2019 Jul 17]; 9(9). Available from: https://www.ncbi.n/m.nih.gov/pmc/articles/ PMC4161416/

18. Sanders $M H$, Newman $A B$, Haggerty $C L$, Redline S, Lebowitz M, Samet J et al (2003) Sleep and sleep-disordered breathing in adults with predominantly mild obstructive airway disease. Am J Respir Crit Care Med. 167(1):7-14

19. Bednarek M, Plywaczewski R, Jonczak L, Zielinski J (2005) There is no relationship between chronic obstructive pulmonary disease and obstructive sleep apnea syndrome: a population study. Respiration. 72(2): 142-149

20. Choi K-M, Thomas RJ, Kim J, Lee SK, Yoon DW, Shin C (2017) Overlap syndrome of COPD and OSA in Koreans. Medicine (Baltimore). 96(27):e7241

21. Sun W-L, Wang J-L, Jia G-H, Mi W-J, Liao Y-X, Huang Y-W et al (2019) Impact of obstructive sleep apnea on pulmonary hypertension in patients with chronic obstructive pulmonary disease. Chin Med J. 132(11):1272-1282

22. Krachman SL, Tiwari R, Vega ME, Yu D, Soler X, Jaffe F et al (2016) Effect of emphysema severity on the apnea-hypopnea index in smokers with obstructive sleep apnea. Ann Am Thorac Soc. 13(7):1129-1135
23. Shiina K, Tomiyama H, Takata Y, Yoshida M, Kato K, Nishihata Y et al (2012) Overlap syndrome: additive effects of COPD on the cardiovascular damages in patients with OSA. Respir Med. 106(9):1335-1341

24. Esmaeel H, Mohammadien H, Saleh A-E, Mohamed F (2019) Prepolysomnography evaluation can predict obstructive sleep apnea and is correlated to its severity. Egypt J BrSonchology. 13:556

25. Sweed RA, Hassan S, ElWahab NHA, Aref SR, Mahmoud MI (2019) Comorbidities associated with obstructive sleep apnea: a retrospective Egyptian study on 244 patients. Sleep Breath. 23(4):1079-1085

26. McNicholas WT, Verbraecken J, Marin JM (2013) Sleep disorders in COPD: the forgotten dimension. Eur Respir Rev. 22(129):365-375

27. Mohsenin V (2005) Sleep in chronic obstructive pulmonary disease. Semin Respir Crit Care Med. 26(1):109-116

28. Collop N (2010) Sleep and sleep disorders in chronic obstructive pulmonary disease. Respiration. 80(1):78-86

29. Redolfi S, Yumino D, Ruttanaumpawan P, Yau B, Su M-C, Lam J et al (2009) Relationship between overnight rostral fluid shift and Obstructive Sleep Apnea in nonobese men. Am J Respir Crit Care Med. 179(3):241-246

30. Singh S, Kaur H, Singh S, Khawaja I (2018) The Overlap Syndrome. Cureus. 10(10):e3453

31. Ryan S, Taylor CT, McNicholas WT (2005) Selective activation of inflammatory pathways by intermittent hypoxia in obstructive sleep apnea syndrome. Circulation. 112(17):2660-2667

32. Yokoe T, Minoguchi K, Matsuo H, Oda N, Minoguchi H, Yoshino G et al (2003) Elevated levels of C-reactive protein and interleukin-6 in patients with obstructive sleep apnea syndrome are decreased by nasal continuous positive airway pressure. Circulation. 107(8):1129-1134

33. Yanbaeva DG, Dentener MA, Creutzberg EC, Wesseling G, Wouters EFM (2007) Systemic effects of smoking. Chest. 131(5):1557-1566

34. Lavie $L$ (2003) Obstructive sleep apnoea syndrome-an oxidative stress disorder. Sleep Med Rev. 7(1):35-51

35. Dröge W (2002) Free radicals in the physiological control of cell function. Physiol Rev. 82(1):47-95

36. Fletcher EC, Schaaf JW, Miller J, Fletcher JG (1987) Long-term cardiopulmonary sequelae in patients with sleep apnea and chronic lung disease. Am Rev Respir Dis. 135(3):525-533

37. Taranto-Montemurro L, Messineo L, Perger E, Salameh M, Pini L, Corda L et al (2016) Cardiac sympathetic hyperactivity in patients with chronic obstructive pulmonary disease and obstructive sleep apnea. COPD. 13(6): 706-711

38. Du W, Liu J, Zhou J, Ye D, OuYang Y, Deng Q (2018) Obstructive sleep apnea, COPD, the overlap syndrome, and mortality: results from the 20052008 National Health and Nutrition Examination Survey. Int J Chron Obstruct Pulmon Dis. 13:665-674

39. Lewis CA, Fergusson W, Eaton T, Zeng I, Kolbe J (2009) Isolated nocturnal desaturation in COPD: prevalence and impact on quality of life and sleep. Thorax. 64(2):133-138

40. McNicholas WT (2016) Chronic obstructive pulmonary disease and obstructive sleep apnoea-the overlap syndrome. J Thorac Dis. 8(2):236242

41. MCNicholas WT (2000) Impact of sleep in COPD. Chest. 117(2 Suppl):48S$53 \mathrm{~S}$

42. Biswas D, Mukherjee S, Chakroborty R, Chatterjee S, Rath S, Das R et al (2017) Occurrence of anxiety and depression among stable COPD patients and its impact on functional capability. J Clin Diagn Res 11(2):OC24-OC27

43. Agusti A, Hedner J, Marin JM, Barbé F, Cazzola M, Rennard S (2011) Nighttime symptoms: a forgotten dimension of COPD. Eur Respir Rev. 20(121): 183-194

44. Donaldson GC, Seemungal TA, Bhowmik A, Wedzicha JA (2002) Relationship between exacerbation frequency and lung function decline in chronic obstructive pulmonary disease. Thorax. 57(10):847-852

45. Soler X, Diaz-Piedra C, Ries AL (2013) Pulmonary rehabilitation improves sleep quality in chronic lung disease. COPD. 10(2):156-163

46. Donovan LM, Feemster LC, Udris EM, Griffith MF, Spece LJ, Palen BN et al (2019) Poor outcomes among patients with chronic obstructive pulmonary disease with higher risk for undiagnosed obstructive sleep apnea in the LOTT cohort. JCSM. 15(01):71-77

47. Jaoude P, El-Solh AA (2019) Predictive factors for COPD exacerbations and mortality in patients with overlap syndrome. Clin Respir J. 13(10):643-651

48. Ordronneau J, Chollet S, Nogues B, Chailleux E (1994) Sleep apnea syndrome in intensive care. Rev Mal Respir. 11(1):51-55 
49. Hiestand D, Phillips B (2008) The overlap syndrome: chronic obstructive pulmonary disease and obstructive sleep apnea. Crit Care Clin. 24(3):551563 vii

50. Marin JM, Soriano JB, Carrizo SJ, Boldova A, Celli BR (2010) Outcomes in patients with chronic obstructive pulmonary disease and obstructive sleep apnea: the overlap syndrome. Am J Respir Crit Care Med. 182(3):325-331

51. Hawryłkiewicz I, Sliwinski P, Górecka D, Pływaczewski R, Zieliński J (2003) Pulmonary haemodynamics in patients with OSAS or an overlap syndrome. Monaldi archives for chest disease $=$ Archivio Monaldi per le malattie del torace / Fondazione clinica del lavoro, IRCCS [and] Istituto di clinica tisiologica e malattie apparato respiratorio, Università di Napoli. Secondo ateneo. 61:148-152

52. Chaouat A, Weitzenblum E, Krieger J, Ifoundza T, Oswald M, Kessler R (1995) Association of chronic obstructive pulmonary disease and sleep apnea syndrome. Am J Respir Crit Care Med. 151(1):82-86

53. Ganga HV, Nair SU, Puppala VK, Miller WL (2013) Risk of new-onset atrial fibrillation in elderly patients with the overlap syndrome: a retrospective cohort study. J Geriatr Cardiol. 10(2):129-134

54. Lahousse L, Niemeijer MN, van den Berg ME, Rijnbeek PR, Joos GF, Hofman A et al (2015) Chronic obstructive pulmonary disease and sudden cardiac death: the Rotterdam study. Eur Heart J. 36(27):1754-1761

55. Jen R, Li Y, Owens RL, Malhotra A (2016) Sleep in chronic obstructive pulmonary disease: evidence gaps and challenges. Can Respir J. 2016: 7947198

56. Lacasse Y, Sériès F, Vujovic-Zotovic N, Goldstein R, Bourbeau J, Lecours R et al (2011) Evaluating nocturnal oxygen desaturation in COPD—revised. Respir Med. 105(9):1331-1337

57. Pronzato C. Chronic obstructive pulmonary disease and obstructive sleep apnea. association, consequences and treatment. 1 [Internet]. 2010 [cited 2019 Aug 6];73(4). Available from: https:/www.monaldi-archives.org/index. $\mathrm{php} / \mathrm{macd} / \mathrm{article} / \mathrm{view} / 285$

58. Celli BR, MacNee W, Task Force ATS/ERS (2004) Standards for the diagnosis and treatment of patients with COPD: a summary of the ATS/ERS position paper. Eur Respir J. 23(6):932-946

59. McNicholas WT (2017) COPD-OSA overlap syndrome: evolving evidence regarding epidemiology, clinical consequences, and management. CHEST. 152(6):1318-1326

60. Soler X, Gaio E, Powell FL, Ramsdell JW, Loredo JS, Malhotra A et al (2015) High Prevalence of obstructive sleep apnea in patients with moderate to severe chronic obstructive pulmonary disease. Annals ATS. 12(8):1219-1225

61. Branson RD (2018) Oxygen therapy in COPD. Respir Care. 63(6):734-748

62. Loredo JS, Ancoli-Israel S, Kim E-J, Lim WJ, Dimsdale JE (2006) Effect of continuous positive airway pressure versus supplemental oxygen on sleep quality in obstructive sleep apnea: a placebo-CPAP-controlled study. Sleep. 29(4):564-571

63. Machado M-CL, Vollmer WM, Togeiro SM, Bilderback AL, Oliveira M-VC, Leitão FS et al (2010) CPAP and survival in moderate-to-severe obstructive sleep apnoea syndrome and hypoxaemic COPD. Eur Respir J. 35(1):132-137

64. de Miguel J, Cabello J, Sánchez-Alarcos JMF, Alvarez-Sala R, Espinós D, Alvarez-Sala JL (2002) Long-term effects of treatment with nasal continuous positive airway pressure on lung function in patients with overlap syndrome. Sleep Breath. 6(1):3-10

65. Mansfield D, Naughton MT (1999) Effects of continuous positive airway pressure on lung function in patients with chronic obstructive pulmonary disease and sleep disordered breathing. Respirology. 4(4):365-370

66. Nowiński A, Bieleń P, Jonczak L, Sliwiński P (2007) Influence of treatment with continuous positive airway pressure on respiratory muscle function and physical fitness in patients with obstructive sleep apnoea and overlap syndrome. Pneumonol Alergol Pol. 75(1):46-56

67. Marin JM, Carrizo SJ, Vicente E, Agusti AGN (2005) Long-term cardiovascular outcomes in men with obstructive sleep apnoea-hypopnoea with or without treatment with continuous positive airway pressure: an observational study. Lancet. 365(9464):1046-1053

68. Mermigkis C, Kopanakis A, Foldvary-Schaefer N, Golish J, Polychronopoulos V, Schiza S et al (2007) Health-related quality of life in patients with obstructive sleep apnoea and chronic obstructive pulmonary disease (overlap syndrome). Int J Clin Pract. 61(2):207-211

\section{Publisher's Note}

Springer Nature remains neutral with regard to jurisdictional claims in published maps and institutional affiliations.

\section{Submit your manuscript to a SpringerOpen ${ }^{\circ}$ journal and benefit from:}

- Convenient online submission

- Rigorous peer review

- Open access: articles freely available online

- High visibility within the field

- Retaining the copyright to your article

Submit your next manuscript at $\boldsymbol{\nabla}$ springeropen.com 